\title{
A COHORT STUDY OF COMORBIDITIES IN COELIAC DISEASE FROM A SINGLE TERTIARY PAEDIATRIC CENTER
}

\author{
Kontaki E, Mitrogiorgou M, Georgantzi G, Attilakos A, Papaevangelou V, Fessatou S
}

Third Department of Paediatrics, National and Kapodistrian University of Athens, University General Hospital "Attikon"

\section{INTRODUCTION}

- Coeliac disease (CD) is an immune-mediated enteropathy that develops in genetically susceptible in dividuals in response to the consumption of gluten.

- CD entails a broad spectrum of clinical manifestations and associated comorbidities.

- In many cases, patients have no gastrointestinal disorders and are referred to paediatric gastroenterologist due to combined endocrine disorders.

- Many patients are under-diagnosed.

\section{OBJECTIVES}

- We aimed to study children with $C D$ and determine the prevalence of paediatric endocrinologists' referrals due to positive antitransglutaminase antibodies (t-TG) and coexisting type 1 diabetes (T1D), autoimmune thyroiditis, or Down syndrome.

- Data were retrospectively reviewed.

\section{CONCLUSIONS}

$>$ Paediatric patients referred from paediatric endocrinologists should be closely monitored for CD in order to allow early diagnosis and treatment onset with gluten-free diet.

$>$ Future studies are warranted in order to further elucidate the association of several controversial comorbidities of $C D$, including EoE.

$>\quad$ Recommendations for screening considerations among CD patients in a cost effective, timely, and efficient manner are necessary as to optimize the prognosis of the disease. Especially, it could be recommended to follow-up children with EoE more closely for CD.

1. Majsiak E et al. [Celiac disease - disease of children and adults: symptoms, disease complications, risk groups and comorbidities]. Pol Merkur Lekarski. 2018

2. Lundin KE et al. Coeliac disease and autoimmune disease-genetic overlap and screening. Nat Rev Gastroenterol Hepatol. 2015

3. Prinzbach A et al. Comorbidities in Childhood Celiac Disease: A Phenome Wide Association Study using the Electronic Health Record. J Pediatr Gastroenterol Nutr. 2018

4. Hommeida S et al. The Association Between Celiac Disease and Eosinophilic Esophagitis: Mayo Experience and Meta-analysis of the Literature. J Pediatr Gastroenterol Nutr. 2017

\section{RESULTS}

- A total of 62 patients were retrieved.

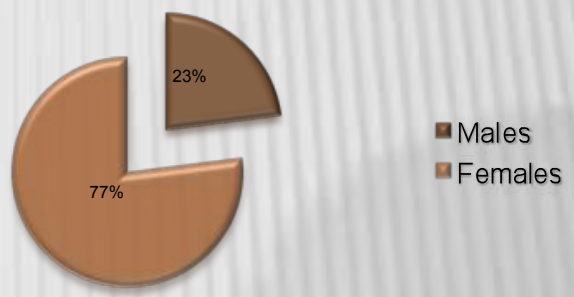

- The median age at diagnosis was 9 years (range: $2-17$ years).

The most common clinical manifestations of patients with CD

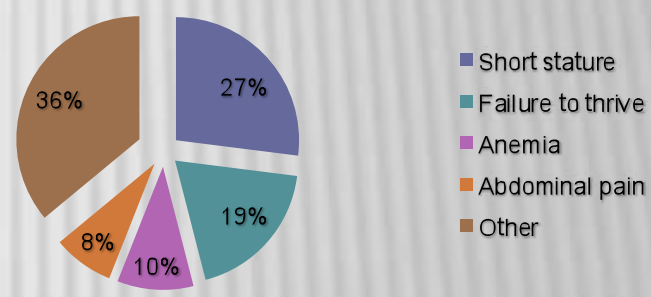

The most prevalent comorbid disorders in patients with CD

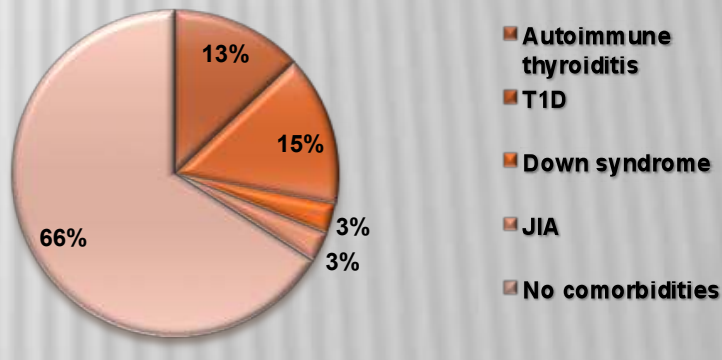

- Among 62 patients, 25 (40\%) were referred from paediatric en docrinologists.

- All children with T1D, abnormal t-TG IgA and HLA DQ2/DQ8 genotype underwent an upper GI endoscopy including Marsh classification in case of CD.

- $42 \%$ of biopsies were consistent with Marsh type IIIB, whereas $5 \%$ of patients were identified with eosinophilic esophagitis (EoE). 